\title{
Weld Seam Recognition Based On CCD Image Processing
}

\author{
Jintao $\mathrm{Yu}^{1}$, Xian $\mathrm{Li}^{2}$, Mingli Ding ${ }^{2}$, Shicheng Dong ${ }^{1}$, Junling $\mathrm{Li}^{1}$ \\ 1. School of Computer and Information Engineering, Harbin University of \\ Commerce, Harbin, China 150028 \\ 2. Dept. of Automatic Test and Control, Harbin Institute of Technology, Harbin, \\ China 150001 \\ hityjt@sina.com
}

\begin{abstract}
Weld seam recognition is the key technique in visual tracking research. Based on the weld image features, the image preprocessing and the linear feature extraction method of the weld seam recognition are designed. The extended adaptive median filtering method and modified Otsu adaptive threshold selection method for image segmentation are used in the image pre-processing process, to remove the noise and compress the image data. Through comparing with several other edge operators, the Roberts operator is chosen to achieve the goal of detecting the welding edge. Finally, the weld seam information is extracted through Hough transform and a satisfied result can be acquired. The study provides a theoretical support for the following designing of the weld tracking system.
\end{abstract}

Keywords: weld recognition, adaptive median filter, image segmentation, edge detection, Hough transform

\section{Introduction}

Welding is one of the most important processes in manufacturing industry. In order to improve welding quality, enhance production efficiency and save human resource, realize automatic industry without human operator, people investigate various methods to achieve the welding process automation and intelligence around the world in the past decades. Weld seam tracking technology is an important research area in automation and artificial intelligence. The advanced weld seam tracking technology can improve welding quality as well as operation safety and economy.

In the weld seam tracking processing, the weld seam identification is one of the important parts. The weld seam identification based on image processing which is broadly concerned by many researchers around the world. There are many research results on weld seam edge exaction based on gray gradient mutation, such as the pool edge extraction [1], The study of the weld seam recognition based on texture feature [2] and mathematical morphology [3] are also going on.

For the sake of extracting the weld feature information in front of welding gun when the gun is firing, in this paper, a weld seam recognition method based on CCD image processing is proposed. The seam recognition algorithm is realized by $\mathrm{VC}++$ software and the experiment results show the correctness of the algorithm.

\section{Weld Seam Identification Method}

Digital image processing methods can be divided into two categories: spatial domain and frequency domain. Among them, the spatial domain method is based on pixel processing from image; frequency domain method is based on modifying the value of Fourier Transform of the original image. The frequency domain methods sometimes can 
not satisfy the speed requirements of real-time image processing; in this paper the spatial domain method is used to imply the weld seam identification as shown in Figure 1.

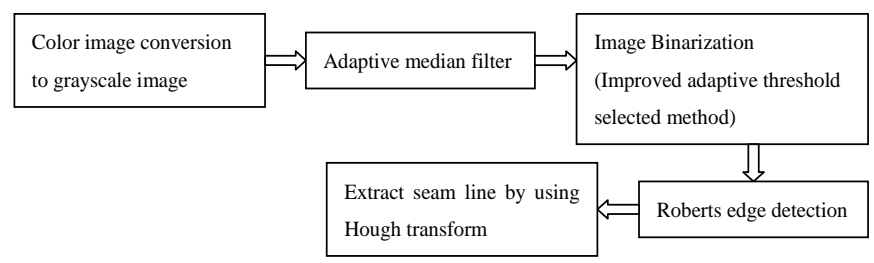

Figure 1. Weld Seam Identification Process

\subsection{Weld Seam Image Preprocessing Methods}

The sampled images are always large and contain much unnecessary information, the processing data should be determined before processing the image.In this paper, the Open Window method is selected to do seam welding processing at un-seamed part, which significantly improves the image processing speed, also reduces the interferences from scene information. CCD visual sensor collects color images and the color image calculation is three times more than gray-scale image. So extracting the weld seam information from the color space cannot meet real time image processing speed requirement. We need converse the color images to the grayscale ones before using the Open Window method. The conversion relation is [4]

$$
\operatorname{Gray}(i, j)=0.11 R(i, j)+0.59 G(i, j)+0.3 B(i, j),
$$

Where $\operatorname{Gray}(i, j)$ is the gray value of the gray-scale image at point $(i, j) ; \mathrm{R}(\mathrm{i}, \mathrm{j}), \mathrm{G}(\mathrm{i}, \mathrm{j})$ and $\mathrm{B}(\mathrm{i}, \mathrm{j})$ are values of red, green and blue component in the original color image, respectively. The transformed gray-scale weld seam image is shown in Fig.2.

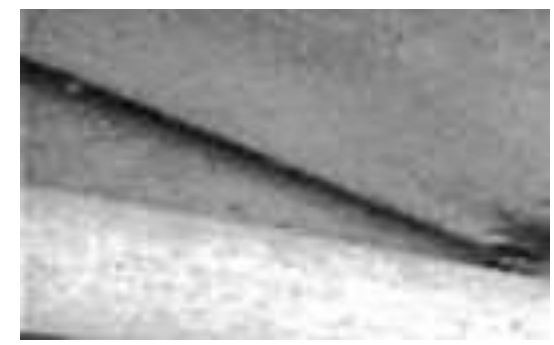

\section{Figure 2. Transformed Grayscale Image of the Weld}

A modified adaptive median filter is proposed here to filter the images. The adaptive median filter is similar as the traditional median filter which produces a rectangular area. The difference between the two methods is that, in the filtering process, the adaptive filter changes the filter window size based on $\mathrm{s}$ certain conditions. The adaptive median filtering method has three characteristics: Firstly, it can remove the salt and pepper (impulse) noise; secondly, in this method, the other non-pulse type noise plays a role of smoothing step; last, this method can reduce the distortion caused by traditional median filter. For example, the objects has too thin or too thick edge phenomenon.

The adaptive median filter algorithm is as follows [5], let

$s_{x y}$ - a sub-image whose center is at $(x, y)$;

$z_{\min }$ - the minimum gray value in $s_{x y}$;

$z_{\max }$ - the maximum gray value in $s_{x y}$;

$z_{\mathrm{m} e d}$-The median of the gray value in $s_{x y}$;

$z_{x y}$ - Coordinates of the gray value; 
$s_{\max }$ - The maximum allowed adaptive median filter window size.

The adaptive median filter algorithm working in two levels, expressed as a level $\mathrm{A}$ and level B:

level A: $A_{1}=z_{\text {med }}-z_{\min }, A_{2}=z_{\mathrm{med}}-z_{\max }$, if $A_{1}>0$ and $A_{2}<0$, turn to level $\mathrm{B}$, otherwise increase window size, if window size $s_{x y} \leq s_{\max }$, repeat level A, otherwise output $z_{x y}$;

Level B: $B_{1}=z_{x y}-z_{\min }, B_{2}=z_{x y}-z_{\max }$, if $B_{1}>0$ and $B_{2}<0$ output $z_{x y}$,otherwise output $z_{\mathrm{med}} \cdot$

Level $\mathrm{A}$ is used to determines the output $z_{\mathrm{med}}$ is noise points or not for the filter. If $z_{\min }<z_{\mathrm{med}}<z_{\max }$ success, due to the pixels value of the noise is the highest point or the minimum pixel value, so that $z_{\mathrm{med}}$ can not be the noise point, so turn to level $\mathrm{B}$. Level B is used to determine if the current point (centre point of filter window) $z_{x y}$ is a pulse point or not. If condition $z_{\min }<z_{x y}<z_{\max }$ success, for the same reason, $z_{x y}$ is not a pulse point as well. In this case, the adaptive median filter algorithm does not change gray value of the current point. This mechanism does not change the current pixel value and greatly reduce the distortion of the image.

If condition $z_{\min }<z_{x y}<z_{\max }$ unsuccessful, nor $z_{x y}=z_{\min }$ or $z_{x y}=z_{\max }$, in the both cases,

the pixels value is an extreme value and $z_{\text {med }}$ is not a noise point based on level A. The adaptive median filter algorithm put the median value of the filter window as the current pixel gray value.

In the above adaptive median filter algorithm, due to $A_{1}=z_{\text {med }}-z_{\text {min }}, A_{2}=z_{\text {med }}-z_{\text {max }}$, So $A_{1}>0$ and $A_{2}<0$. This method actually determines whether $z_{\min }, z_{\text {med }}$ and $z_{\max }$ are equal. If they are equal, the method increases the filter window. When the size of the filter window reaches to the maximum value, $z_{\min }, z_{\text {med }}$ and $z_{\max }$ still are equal, the algorithm outputs the current value.

In response to these deficiencies, the adaptive median filter is improved. This extended adaptive median filter is modified at determine conditions: $A_{1}=z_{\text {med }}-z_{\min }-1$ , $A_{2}=z_{\text {med }}-z_{\max }+1 ; B_{1}=z_{x y}-z_{\min }-1, B_{2}=z_{x y}-z_{\max }+1$. This configuration deletes the value of $z_{\text {min }}=z_{\text {med }}=z_{\text {max }}, z_{\text {min }}+1=z_{\text {med }}=z_{\text {max }}, z_{\text {min }}=z_{\text {med }}=z_{\text {max }}-1, z_{\text {min }}+1=z_{\text {med }}=z_{\text {max }}-1$ is deemed as noise. Two images processed after the median filter and adaptive median filter are shown in Figure 3a) and Figure 3b). Looking to the results, there are less image noise by using adaptive median filter.

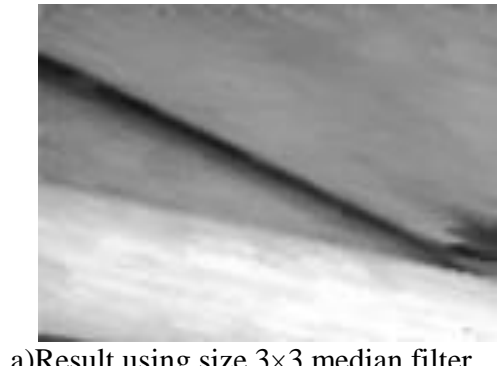

a)Result using size $3 \times 3$ median filter

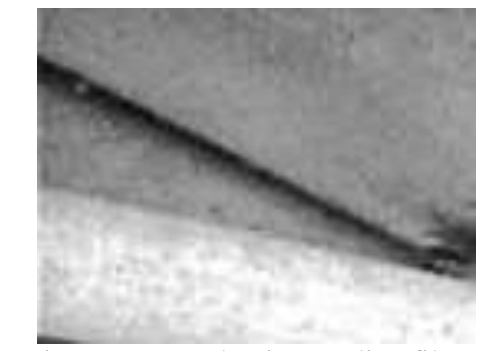

b)Using $\operatorname{smax}=7$ adaptive median filter

Figure 3. Results of Image Filtering

\subsection{Weld Seam Image Segmentation}

Threshold segmentation is a typical image segmentation method. This method is setup based on the gray-scale distribution of an image before processing it. The value of grayscale threshold $\mathrm{T}$ is the key issues on image segmentation. Too big or too small of value $\mathrm{T}$ cause the error on the size and the shape of the object after segmentation. The histogram 
figure after filtering of weld image is shown in Figure 4. Looking to Figure 3b), the grayscale level value of the weld higher than the background, a gray level threshold value can be chosen as the key value according to Figure 4 to separate the weld line from the background. Because the real-time gray scale image contains an inconstant background value, and the steel gray value changes, a good threshold value of an image maybe unworkable in other images. In this paper, the adaptive threshold selection method-Otsu method is selected to conquer this problem mentioned above. This paper also improved its algorithm based on the principle.

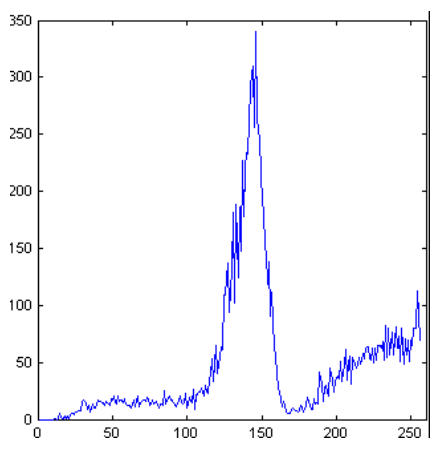

Figure 4. Weld Seam Image's Histogram after Filtering

The Otsu adaptive threshold segmentation ${ }^{[6]}$ principles as follows: Assuming a gray image is described in a two dimensional matrix $F_{M \times N}=[f(x, y)]_{M \times N}, M \times N$ is the size of the image, $f(x, y)$ is the gray value of image $(x, y)$ and $f(x, y) \in\{0,1, \ldots, L-1\}, L$ is sum of gray level. If the appearance times of gray level $i$ in the image is $n_{i}$, the probability of gray level $i$ is

$$
p_{i}=\frac{n_{i}}{M \times N}, \quad \text { and } p_{i} \geq 0, \quad \sum_{i=0}^{L-1} p_{i}=1 .
$$

If all pixels are divided into two class by gray-level $\mathrm{T}$ : $\mathrm{S} 1$ includes pixels of $i \leq T, \mathrm{~S} 2$ includes pixels of $i>T$. The probabilities of $\mathrm{S} 1$ and $\mathrm{S} 2$ are

$$
P_{1}=\sum_{i=0}^{T} p_{i}, P_{2}=\sum_{i=T+1}^{L-1} p_{i}, P_{1}+P_{2}=1 .
$$

The centre class of S1and S2 are

$$
\omega_{1}=\sum_{i=0}^{T} i p_{i}, \omega_{2}=\sum_{i=T+1}^{L-1} i p_{i}
$$

and

$$
P_{1} \omega_{1}+P_{2} \omega_{2}=\omega_{0}=\sum_{i=0}^{L-1} i p_{i}
$$

The between-class variance S1and $\mathrm{S} 2$ is

$$
\sigma^{2}=P_{1}\left(\omega_{1}-\omega_{0}\right)^{2}+P_{2}\left(\omega_{2}-\omega_{0}\right)^{2}=P_{1} P_{2}\left(\omega_{2}-\omega_{1}\right)^{2} .
$$

Obviously, $P_{1}, P_{2}, \omega_{1}, \omega_{2}$ and $\sigma^{2}$ are the functions of gray threshold value $T$.

To achieve the optimal image segmentation, Otsu let two class's between-class variance as a function of the threshold identification and consider that the optimal threshold value $T^{*}$ is the gray-scale value which makes $\sigma^{2}$ maximal. The optimal discriminate threshold is

$$
\sigma^{2}\left(T^{*}\right)=\max \sigma^{2}(T) .
$$

The Otsu threshold selection method is used to choose the threshold value on the weld seam image segmentation. The result is shown in Figure 5a) $\left(T^{*}=177\right)$. Obviously, the processed image cannot properly segment the weld and the background. So the threshold 
value of recognition function is changed to achieve the image segmentation properly. In order to fully reflect the merits and shortcomings of classification, achieve more accurate segmentation, the adaptive threshold method re-defines the two class distance and introduces the dispersion of the class.

As mentioned above, (2) (4) gives the class center within the class S1 and S2. In order to describe the distance between the two classes effectively, define the distance of two classes

$$
D=\left|\omega_{1}-\omega_{2}\right| .
$$

In (8), D somewhat reflects the classification effect of S1 and S2. If D is larger, it means that the distance between the two classes is larger. In addition, in class S1 and class $\mathrm{S} 2$, the good or bad of cohesion is an important symbol reflection of the effectiveness of the classification directly. In S1 and S2, the class of dispersion is defines as the distance from each pixel to the centre within the class, like

$$
\begin{array}{r}
d_{1}=\sum_{i=0}^{T}\left|i-\omega_{1}\right| * p_{i}, \\
d_{2}=\sum_{i=T+1}^{L-1}\left|i-\omega_{2}\right| * p_{i},
\end{array}
$$

Obviously, the smaller the dispersion of each class, the better the cohesion as well as the classification.

Considering the two factors above, to ensure a good classification results, it should let $\mathrm{D}$ maximum and $\mathrm{d} 1, \mathrm{~d} 2$ minimum. In this way, each class has best cohesion and the two classes have longest distance. At this point, two class of the image will be separate to a great extent. Therefore, the threshold value identification function of the segmentation is defined as

$$
H(T)=\frac{P_{1} * P_{2} * D}{d_{1}+d_{2}} .
$$

It can be found that when $\mathrm{H}$ is maximum, the best classification effect, the optimal threshold discriminate as

$$
H\left(T^{*}\right)=H_{\text {max }}(T) .
$$

If the gray level $T^{*}$ of an image can let $H\left(T^{*}\right)=H_{\max }(T)$, and $T^{*}$ is the optimal threshold value, it will divide image $F_{M \times N}=[f(x, y)]_{M \times N}$ into class S1and class S2. At this time, if all pixels gray value in $\mathrm{S} 1$ is set as 255 and all pixels gray value in S2 as 0, the image $F_{M \times N}=[f(x, y)]_{M \times N}$ is segmented. The segmentation process can separate the target from the background maximally and achieve best effect.

The result acquired by using the Otsu threshold selection method to choose the threshold value on the weld seam image segmentation is shown Fig. 5b) $\left(T^{*}=83\right)$. From the figure, it can be found that the procedure separates weld seam form background successfully.

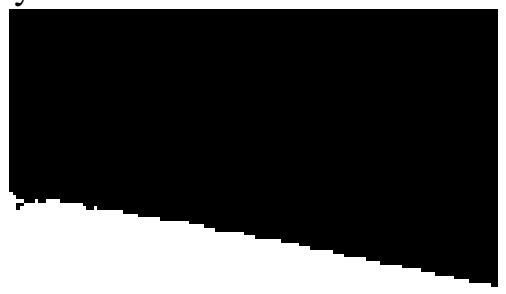

a) Otsu segmentation method

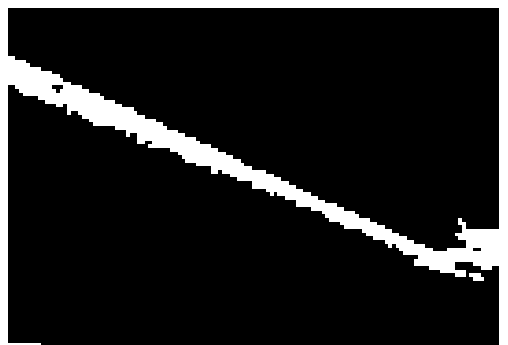

b) Improved Otsu segmentation method

Figure 5. Image after segmentation 


\subsection{Edge Detection and Feature Extraction Method}

The purpose of this paper is to obtain information of seam central. Firstly, we need obtain seam edge feature information, and then extract weld seam line on next processing. Hough transformation is selected on weld seam edge line extraction ${ }^{[7]}$. Hough transformation has the advantage of anti-interference. Even there is disturbance, gap and broken line in the image, after Hough transformation, the clear peaks can be formed in the transform space. The steps of Hough transformation which can be found in many literatures are not described here.

\section{Experiment Results}

The experiments using four kinds of common edge detection algorithms are investigated. The results are shown in Figure 6.

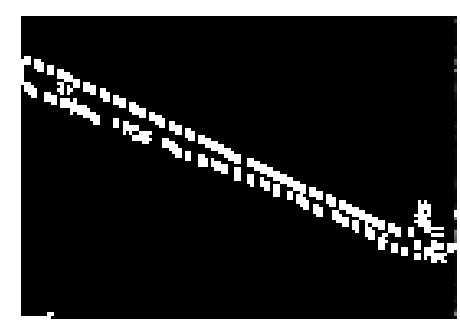

a)Sobel operator

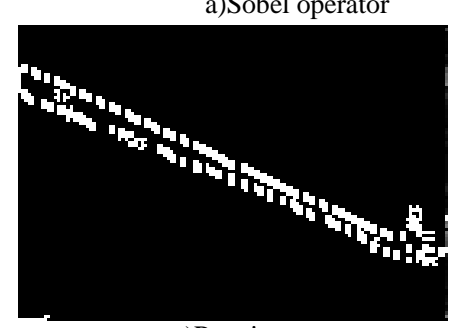

c)Prewitt operator
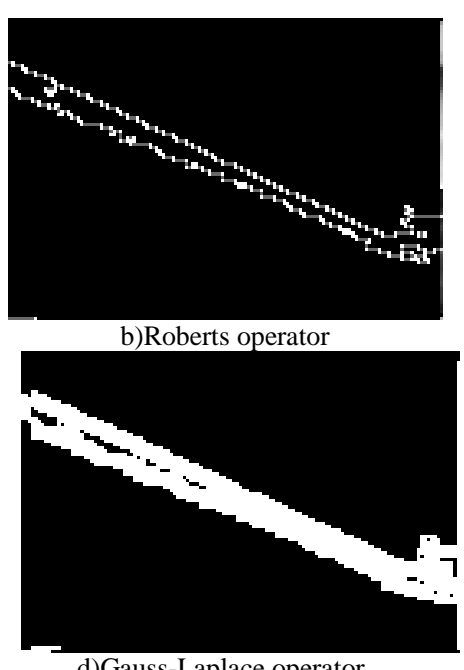

d)Gauss-Laplace operator

Figure 6. Results of Edge Detection

Comparing the four processed image results, it can be found that Roberts operator uses the discrepancy between two adjacent pixels as the approximate gradient magnitude to detect the image edge. This operator has high precision and high sensitivity to noise. Sobel operator detects the edge by adjacent pixels gray-weighted difference to achieve the extreme edge phenomenon. Sobel operator has a smoothing effect on reducing noise and also provides the edge direction information with more accuracy, but the edge of the positioning accuracy is not high enough which is not suitable for the applications with high accuracy demand. Prewitt operator detects edge by adjacent pixels gray difference to achieve extreme edge phenomenon. Prewitt operator has a smoothing effect on reducing noise as well as poor location accuracy character. Gauss-Laplace operator is a second order differential operator, which detects the edge by using the second derivative edge where zero crossing occurs. This operator has no directionality and it cannot get the edge direction information either. It is sensitive to the gray mutation and has a relative low processing speed. Based above, Roberts operator is determined to detect the weld seam edge of the image. The result of Figure 6b) is used to extraction seam as a follow-up purpose. 7.

The result after detecting the edge and extracting the weld seam line is shown in Figure 


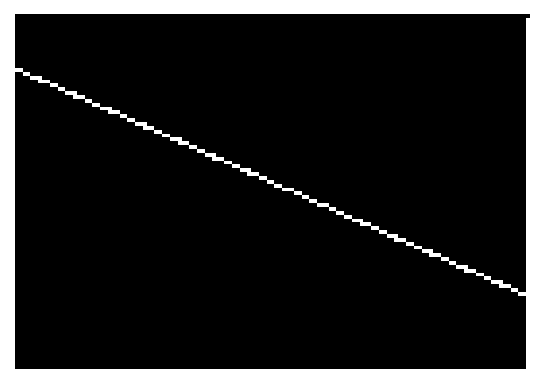

Figure 7. Result after Hough Transformation

Using the direct Hough transform method can detect the weld seam line and the basic principle is same as detecting a single line. The selection of the threshold value on accumulator unit which appears on partial peaks is a little difference in this paper. After many trials, $1 / 3$ of partial peak is selected as the best threshold value.

\section{Conclusions}

The weld seam identification method on image processing is discussed in this paper. The image preprocessing procedure, including the seam edge detection and the seam recognition of the image that based the seam line feature extraction, is also designed. The experiment results show the feature information of the weld seam line extracted successfully, that achieve the weld seam identification purpose successfully.

\section{Acknowledgements}

This work is supported by the science and technology research Foundation of Heilongjiang Education Department (Grant No. 12531148), the National Natural Science Foundation of China (Grant No. 61171196), the Program of Research and Development of Applied Technology of Heilongjiang Province (China) (Grants No. GC13A310), and the Research Fund of Harbin (China) innovative talent in science and technology (Grants No. 2013RFLXJ020).

\section{References}

[1] K. Y. Bae, T. H. Lee and K. C. Ahn, "Journal of Material Processing Technology", vol. 120, no. 3 (2002).

[2] S. Wang, D. Du, K. Zeng and Y. Zou, "Transactions of the China Welding Institution”, vol. 29, no. 11, (2008).

[3] J.-Q. Shen, S.-s. Hu, S.-q. Feng and L.-n. Zhu, "Journal of Tianjin University”, vol. 43, no. 4, (2010).

[4] X.-Q. Zhou and X.H. Liu, "Techniques of Automation and Applications", vol. 24, no. 3, (2005).

[5] J. Wang, C. P. Wang and H. T. Jia, "Measurement \& Control Technology", vol. 23, no. 5, (2004).

[6] M. Chen, "Computer Applications and Software", vol. 23, no. 4, (2006).

[7] W. Qu, Y. Zhang and G. Yan, "Chinese Journal of Mechanical Engineering", vol. 41, no. 9, (2005).

\section{Author}

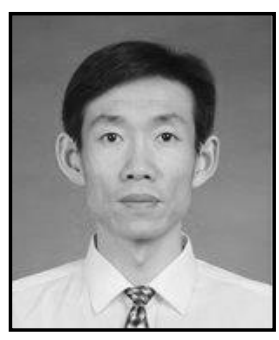

Jintao Yu, Doctor, associate professor, Director of electronic and information engineering. Main research fields include signal processing of sensor, fault detection, wireless sensor networks etc. 
International Journal of Signal Processing, Image Processing and Pattern Recognition Vol.8, No.6 (2015) 